\title{
Effects of oral methadone consumption on visual discrimination performance of the rhesus monkey*
}

\author{
KRISTIN R. CARLSON $\dagger$ and MARIANNA PAVSEK \\ Department of Pharmacology, School of Medicine, University of Pittsburgh, Pittsburgh, Pennsylvania 15261
}

\begin{abstract}
Monkeys which drank small doses, once daily, of methadone mixed with Tang orange drink were trained to perform a series of two-object visual discrimination problems. Their average number of trials to criterion was the same as that of normal monkeys, and they made the same number of errors on test trials in which the orientation of the stimulus objects was changed. Their average choice latencies, however, were significantly longer than those shown by the normal group. In a second experiment, on each trial Ss were given a decision interval to observe the stimulus objects. Latencies, as measured from the end of the interval, were the same for control and methadone-consuming monkeys. These data suggest that the previously elevated latencies were not due to motor or motivational lethargy, but to a drug-induced prolongation of the time necessary to decide which was the correct object.
\end{abstract}

Prior investigations of the behavioral effects of narcotic analgesics have focused on changes in well-established operant responses as a consequence of drug administration. In the monkey, and other species, a general finding has been a depression in response rate after injection of morphine (Bigelow \& Thompson, 1971; Carney, 1973; Hill et al, 1957; Holtzmann \& Villarreal, 1969, 1973; McMillan \& Morse, 1967; Molinego, 1964; Thompson et al, 1970) and methadone (Bigelow \& Thompson, 1971; McMillan et al, 1970). These results suggest that facility in emitting responses is impaired by acute administration of narcotic drugs, through motor lethargy or motivational indifference.

We approached the general topic of behavioral effects of drug consumption somewhat differently. First, we were interested in narcotic drug action on the acquisition of information and skills, rather than on the performance of well-learned behavior. Second, we decided to study behavior more complex than operant responding. We were interested in the monkey's ability to solve relatively demanding problems, to recognize stimulus objects after their orientation was changed, and to deal with a long series of different problems. Finally, since meeting these objectives necessitated chronic drug treatment, we chose to use methadone which was self-administered orally, in subdependence-producing doses. We felt that by limiting the Ss' drug intake, we could avoid gross motor impairment and perhaps reveal more subtle deficits in behavioral processes.

Thus, in Experiment I, we administered a set of visual discrimination problems, and compared the performance of methadone-consuming monkeys to that of normal monkeys reported in an earlier paper (Carlson, 1973).

*Supported by Grant MH20121 from the National Institute of Mental Health to K.R.C. The excellent technical assistance of K. Erleba, M. Gasior, C. Krug, and V. Trammell is gratefully acknowledged. Part of this research was briefly summarized in the Narcotic Antagonist Research Review, 1973, 1, No. 3, p. 4. tSend requests for reprints to K. R. Carlson, 620 Scaife Hall, University of Pittsburgh, Pittsburgh, Pennsylvania 15261.
We observed a difference in behavior between those groups which could have resulted from either of two factors, and Experiment II was designed to decide between those alternatives.

\section{EXPERIMENT I}

\section{Method}

Subjects. The methadone group consisted of three male rhesus monkeys, Macaca mulatta, approximately 2 years old at the beginning of the study, who had no prior drug or experimental history. They were housed individually, had ad lib access to water from an automatic dispensing system, and were fed once daily in the late afternoon. Ss were weighed twice weekly. From 8 to 9 a.m. every day, a bottle containing a limited amount of methadone hydrochloride (Dolophine $\mathrm{HCl}, 10 \mathrm{mg} / \mathrm{ml}$; Lilly) mixed with $100 \mathrm{ml}$ Tang orange drink was placed on the front of each S's cage. At the time the present discrimination data were being collected, one $\mathrm{S}$ was voluntarily consuming an average of $0.50 \mathrm{mg} / \mathrm{kg}$ body weight per day, the second was consuming $1.30 \mathrm{mg} / \mathrm{kg}$, and the third was consuming $1.45 \mathrm{mg} / \mathrm{kg}$. These doses were not sufficient to produce strong physical dependence, since Ss showed no symptoms to a challenge with $10 \mathrm{mcg} / \mathrm{kg} \mathrm{IM}$ naloxone hydrochloride (Nalline $\mathrm{HCl}, 0.4 \mathrm{mg} / \mathrm{ml}$; Endo).

The normal group consisted of five male rhesus monkeys, of similar age and housed under identical conditions, who were the S population of a previous paper (Carlson, 1973) and whose data are presented again here. They received neither Tang nor methadone.

Procedure. A full description of the apparatus and testing procedure has been published elsewhere (Carlson, 1973). Briefly, it consisted of 30-min training sessions which were given 5 days per week between 1 and 4 p.m. Ss were pretrained (Schrier, $1965)$ to perform two-object visual discriminations in a Wisconsin General Test Apparatus (WGTA). They then solved a series of successive two-object problems between randomly derived geometric figures (A ttneave \& Arnoult, 1956) having 4, $6,8,10,12$, or 14 sides. The figures were equated to a $30 \mathrm{~cm}^{2}$ area and cut from $1 / 2$-in.thick black Plexiglas (see Carlson, 1973, for examples of the random figures). Both objects in each problem had the same number of sides, and were presented in the same orientation on each training trial. Four problems at each sidedness level were presented in random order, and Ss were required to meet a criterion of $36 / 40$ correct on each problem. Latencies were measured by a $1 / 100$-sec stop clock, from the time the opaque screen between $\mathrm{S}$ and the objects was raised to 
Table 1

Discrimination Performance

\begin{tabular}{lrr}
\hline & \multicolumn{2}{c}{ Experimental Groups } \\
\cline { 2 - 3 } & Control* $^{*}$ & Methadone \\
\hline Experiment I & & \\
Mean Trials to Criterion & 40.55 & 37.05 \\
$\quad$ Mean Errors in Rotation Test & 2.83 & 2.86 \\
Experiment II & & \\
Mean Trials to Criterion & 22.75 & 23.14 \\
Mean Errors in Rotation Test & 1.83 & 1.45 \\
\hline
\end{tabular}

*The control group in Experiment I consisted of normal Ss $(N=5)$ and in Experiment II of Tang Ss $(N=2)$.

the time at which $S$ first touched an object. A trial was terminated and scored as an error if $\mathrm{S}$ did not make a choice within $30 \mathrm{sec}$, but these times were not included in the latency data. The intertrial interval was $20 \mathrm{sec}$. After criterion performance was attained on each problem, both stimulus objects were rotated $180 \mathrm{deg}$, and an additional 20 test trials were given, maintaining the same reinforcement contingencies.

\section{Results and Discussion}

Due to the general lack of differences as a function of the number of sides comprising the stimulus objects, data were averaged across all sidedness levels. As shown in the top half of Table 1, the methadone and normal groups did not differ in mean trials necessary to reach criterion (Mann-Whitney $U$ test, $z=0.56$, n.s.), or in mean errors after rotation of the stimulus objects (Mann-Whitney, $\mathrm{z}=0.72$, n.s.). In addition, there was no relationship between trials or errors and methadone dosage. Thus, it appears that chronic low-dose methadone consumption is unrelated to the ability to solve successive discrimination problems and to recognize the stimulus objects after their orientation is changed. Nonetheless, it is possible that this type of problem is too simple to tax even an impaired monkey's intellectual capacity, and that any drug-induced blunting of problem-solving ability would be revealed only with more complex associative tasks. We are currently pursuing this line of investigation using monkeys who consume these and larger doses of methadone.

The latency data shown in the left panel of Fig. 1, however, do reveal differences between the methadone and normal groups. Taking the methadone group as a whole, mean latency, when choosing the correct object, was significantly increased during training (Mann-Whitney, $\mathrm{z}=3.23, \mathrm{p}<.001$ ) and during the rotation test (Mann-Whitney, $\mathrm{z}=3.05, \mathrm{p}<.01$ ). This effect is partially dose-related in that the $\mathrm{S}$ consuming the smallest dose exhibited normal latencies, and the other Ss at higher doses were responsible for the significantly elevated group means. Thus we suggest that even moderate doses of methadone may be sufficient to prolong choice latencies in this type of discrimination task. As to the mechanism by which this effect is produced, there are two immediately apparent alternatives: a prolongation of the time necessary to reach a decision as to which object is correct, or an increase in the time necessary to execute a decision by reaching out to move the correct object, due to motor retardation or indifference about the reward. Experiment II was designed to investigate these alternative explanations.

\section{EXPERIMENT II}

This experiment was essentially a replication of Experiment I, except that on every trial a decision period, during which $\mathrm{S}$ could see the stimulus objects but could not touch them, was given. Latencies were measured from the end of the decision period, when the objects became accessible to S's hands, to the moment at

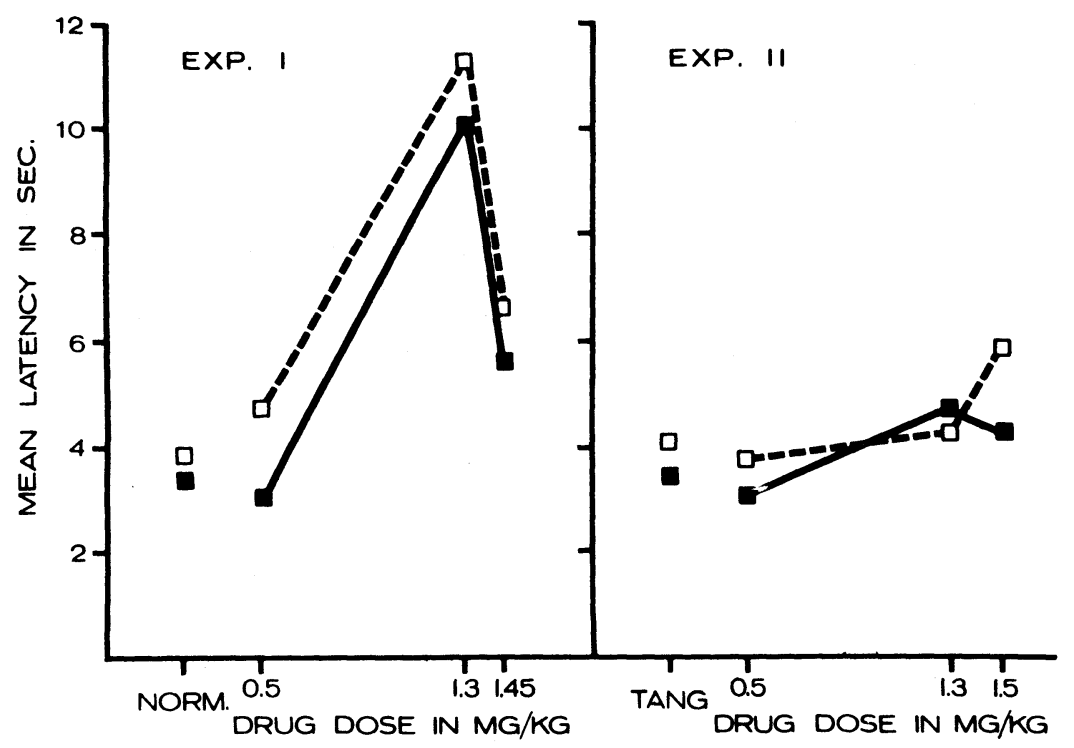

Fig. 1. Mean latency to the correct object in Experiments I and II, for the control Ss and the three Ss consuming methadone. Latencies during training are represented by filled squares and solid lines, and latencies during the rotation test by open squares and dashed lines. 
which he touched one. If the elevated latencies shown by methadone Ss in the prior experiment were due to difficulty in executing a decision, then their latencies should continue to be longer than those of control Ss. If, on the other hand, elevated latencies were derived from difficulty in reaching decisions, then when an adequate amount of time for decision-making is provided, and latencies measured from the end of that period, the latencies of the methadone and control Ss should be equivalent.

\section{Method}

Subjects. The methadone group consisted of the same three Ss as in Experiment I. Their average daily methadone consumption was unaltered except for the $\mathrm{S}$ which rose from 1.45 to $1.50 \mathrm{mg} / \mathrm{kg}$.

The normal Ss of Experiment I were being used in another study (Carlson \& Eibergen, 1974). Therefore, we formed a Tang control group, consisting of two male rhesus monkeys of approximately the same age as the methadone Ss, who had no drug or experimental history. They were treated identically except that no methadone was added to their Tang.

Apparatus. The WGTA used in Experiment I was modified in the following way. The opaque forward screen between $S$ and the stimulus objects was replaced by a screen twice' as high which had an opaque upper half and a transparent Plexiglas lower half. When the screen was in its lowest position S could not see the stimulus objects, when it was raised halfway he could see the objects but could not touch them, and when it was fully raised he had complete access to the objects.

A different series of equal-sided pairs of random figure stimulus objects having $4,6,8,10,12$, or 14 sides was used.

Procedure. The training paradigm was identical to that used before, except for the decision period. To initiate a trial, the forward screen was raised halfway. It was held there for $10 \mathrm{sec}$, and then raised fully. Latencies were measured by a $1 / 100$-sec stop clock, from the end of the $10-\sec$ decision interval to the time $\mathrm{S}$ touched either object.

The methadone $\mathrm{S}$ consuming $0.50 \mathrm{mg} / \mathrm{kg}$ learned three problems at each sided ness level, and all the other Ss learned four problems at each level as in Experiment I.

\section{Results and Discussion}

Since there were no differences as a function of sidedness level of the problems, data from all problems were averaged. The results of Experiment $I$, an absence of between-group differences in trials to criterion and errors after rotation, were replicated in this experiment, as can be seen in the bottom half of Table 1. Mann-Whitney U tests substantiated the lack of differences between methadone and Tang groups in trials $(\mathrm{z}=0.55$, n.s. $)$ and errors $(\mathrm{z}=1.19$, n.s. $)$.

It might be argued that the equivalence of the groups in this experiment actually represents a deficit in the methadone Ss, since they might be expected to exhibit superior performance by virtue of their extensive prior WGTA experience. If such a deficit is produced by methadone, then this effect should be dose-related, higher-dose Ss requiring more trials to criterion and exhibiting more rotation errors. Data from individual Ss are not presented, but we observed such orderly relationships in the opposite direction: as dose increased, both trials and errors decreased. Whatever the basis for this finding, these results do not support the suggestion of any consistent drug-related impairment in performance, as measured by trials to criterion or rotation errors.

When one compares trials and errors between the two experiments, it is clear that the decision period improved the performance of the methadone Ss. The group, as a whole, required significantly fewer trials to criterion with the decision period (Wilcoxon test, $\mathrm{T}=28, \mathrm{p}<.05$ ) and made fewer errors during the rotation test (Wilcoxon, $\mathrm{T}=22.5, \mathrm{p}<.01$ ). Although individual data are not shown, these were consistent findings for all three Ss. It is difficult to attribute this proficiency to increased test sophistication, since the Tang Ss were experimentally naive yet performed as well as the methadone Ss. Furthermore, normal and Tang Ss were equally inexperienced at the beginning of their respective experiments, but the Tang group was significantly superior to the normal group in both trials to criterion (Mann-Whitney, $\mathrm{z}=2.50, \mathrm{p}<.01$ ) and rotation errors (Mann-Whitney, $\mathrm{z}=1.79, \mathrm{p}<.05)$. Thus it does not appear that this phenomenon is related to an S's drug state but, rather, provides experimental confirmation of the widely held belief that observation times improve discrimination performance (Meyer et al, 1965).

The major result of this experiment is depicted in the right panel of Fig. 1. It can be seen that the imposition of a decision interval had no effect on the latencies of control Ss; the Tang group did not differ from the normal group of Experiment $I$ either during training (Mann-Whitney, $\mathrm{z}=0.98$, n.s.) or during the rotation test (Mann-Whitney, $z=0.66$, n.s.). However, the decision time greatly reduced the methadone group's latencies such that they did not differ from the Tang group's during training (Mann-Whitney, $\mathrm{z}=1.57$, n.s.) or during the rotation test (Mann-Whitney, $z=1.36$, n.s.). In addition, there was a significant decrease between Experiments I and II for the methadone group, as a whole, in latencies during training (Wilcoxon, $T=11$, $\mathrm{p}<.005$ ) and during the rotation test (Wilcoxon, $\mathrm{T}=16, \mathrm{p}<.005$ ).

It is interesting that these results were producec almost entirely by changes in the latencies of the two higher-dose methadone Ss. The decision time did not generally reduce latencies, as it had trials to criterion and rotation errors, but specifically affected the latencies of the methadone $\mathrm{Ss}$ in a dose-related fashion. It is irrelevant to this argument whether there is a "floor effect" preventing any S's latency from averaging less than approximately $3 \mathrm{sec}$; the point is that when they were given a decision interval, two formerly slow Ss performed with normal latencies.

It might also be suggested that the decision interval merely served as a warning signal, assisting Ss in orienting themselves toward the stimulus objects and 
thereby reducing latencies. However, for several reasons, we do not favor this explanation. First, we frequently observed that, during the decision interval, Ss would look at the objects and then move to another part of the WGTA. Only when the transparent screen was raised would they move forward and touch an object. Further, in Experiment $I$ there were many other cues indicating that the beginning of a trial was imminent, such as a constant intertrial interval and noises as the stimulus objects and tray were positioned. Thus, in both experiments, the event signaling that a choice could be made was the same-raising the forward screen-and in both cases this event was preceded by warning signals. Nonetheless, only when the objects were visible for an interval before a decision could be executed were the latencies of higher-dose methadone Ss normal.

\section{GENERAL DISCUSSION}

The results of both experiments indicate that moderate doses of methadone have no effect on monkeys' ability to solve successive visual discrimination problems or to recognize stimulus objects when their orientation is changed. However, monkeys consuming $1.30-1.50 \mathrm{mg} / \mathrm{kg}$ methadone do exhibit longer choice latencies. This impairment is not due to motor incoordination or lethargy, since their speed of executing previously made decisions is normal. Rather, we suggest that it is attributable to their need for above-average time to reach decisions.

The finding that response speed was unaltered by methadone is at variance with numerous reports of decreased operant responding after administration of narcotic analgesics (Bigelow \& Thompson, 1971; Carney, 1973; Hill et al, 1957; Holtzman \& Villarreal, 1969, 1973; McMillan \& Morse, 1967; McMillan et al, 1970; Molinego, 1964; Thompson et al, 1970). We believe this discrepancy is a function of dosage; our doses were well below the levels used in other studies, and furthermore, were administered orally-a less effective route than parenterally.

Because we required Ss to learn discrimination problems rather than simply to emit well-practiced operants, we were able to study a process more complex than conventional operant responding. Furthermore, because the methadone dosages were low, we were able to make initial observations of a drug-induced deficit which was not attributable to, or masked by, gross motor impairment. We are attempting to quantify this decision-time deficit by titrating the amount of time Ss have available for decision making according to the accuracy of these decisions. We predict that the latency titration level will be longer for methadone-consuming than for control monkeys.

\section{REFERENCES}

Attneave, F., \& Arnoult, M. The quantitative study of shape and pattern perception. Psychological Bulletin, 1956, 53, 452.471.

Bigelow, G., \& Thom pson, T. Behavioral effects of morphine and methadone in rhesus monkeys. Psychonomic Science, 1971 24, 215-217.

Carlson, $K$. Visual discrimination learning of random figure problems by rhesus monkeys. A nimal Learning \& Behavior, $1973,1,33-37$.

Carlson, K., \& Eibergen, R. Factors influencing the acquisition of tactual random figure discriminations by rhesus monkeys. Animal Learning \& Behavior, 1974, 2, 133-137.

Carney, J. Effects of morphine, codeine and nalox one on foodand codeine-reinforced responding in the rhesus monkey. Federation Proceedings, 1973, 32, 726.

Hill, H., Pescor, H., Belleville, R., \& Wikler, A. Use of differential bar-pressing rates of rats for screening analgesic drugs. I. Techniques and effects of morphine. Journal of Pharmacology \& Experimental Therapeutics, 1957, 120, 388-397.

Holtzman, S., \& Villarreal, J. The effects of morphine on conditioned suppression in rhesus monkeys. Psychonomic Science, 1969, 17, 161-162.

Holtzman, S., \& Villarreal, J. Operant behavior in the morphine-dependent rhesus monkey. Journal of Pharmacology \& Experimental Therapeutics, 1973, 184, 528-541.

McMillan, D., \& Morse, W. Some effects of morphine and morphine antagonists on schedule-controlled behavior. Journal of Pharmacology \& Experimental Therapeutics, 1967, $157,175-184$

McMillan, D., Wolf, P., \& Carchman, R. Antagonism of the behavioral effects of morphine and methadone by narcotic antagonists in the pigeon. Journal of Pharmacology \& Experimental Therapeutics, 1970, 175, 443-458.

Meyer, D., Treichler, F., \& Meyer, P. Discrete-trial training techniques and stimulus variables. In A. Schrier, H. Harlow, and F. Stollnitz, (Eds.), Behavior of non-human primates. Vol. 1. New York: Academic Press, 1965, Pp. 1-49.

Molinego, L. Effects of morphine on the operant behavior of rats. Psychopharmacologia, 1964, 6, 347-367.

Schrier, A. Pretraining performance of three species of macaque monkeys. Psychonomic Science, 1965, 3, 517-518.

Thompson, T., Trombley, J., Luke, D., \& Lott, D. Effects of morphine on behavior maintained by four simple food-reinforcement schedules. Psychopharmacologia, 1970, 17, 182-192.

(R eceived for publication February 12, 1974; revision received A pril 23, 1974.) 